ORIGINAL ARTICLE

\title{
Prevalence of autistic spectrum disorders in Lothian, Scotland: an estimate using the "capture-recapture" technique
}

\author{
M J Harrison, A E O'Hare, H Campbell, A Adamson, J McNeillage
}

Arch Dis Child 2006;91:16-19. doi: 10.1136/adc.2004.049601

See end of article for authors' affiliations

Correspondence to: Correspondence to:
Dr A O'Hare, University of Edinburgh, Department of Child Life and Health, 10 Chalmers Crescent, Edinburgh EN9 1TS, UK; A.O'Hare@ed.ac.uk

Accepted 11 October 2004 Published Online First 26 September 2005

\begin{abstract}
Aims: To determine the prevalence of autistic spectrum disorder in a geographically defined population for children receiving services and compare this to the estimated prevalence based on a two source "capturerecapture" technique previously employed in biological populations to give a "true" prevalence with full ascertainment.

Methods: Information on gender, age, and postcode sector was determined from nine different datasets maintained for children with autistic spectrum disorder and point prevalence was calculated. Data from the diagnostic services and the Special Needs System were entered into the two source "capture-recapture" calculation.

Results: Of a total population of 134661 under 15 year olds resident in Lothian in southeast Scotland, 443 were known to autism services, with a point prevalence of 32.9 per 10000 (95\% Cl 29.8 to 36.0 ). The estimated prevalence using a capture-recapture method was 44.2 (95\% Cl 39.5 to 48.9 ), which suggests that $74 \%$ of affected children were registered with services in some way. The age distribution was similar to that of the background population under the age of 12 years and there was no indication of a rising prevalence. The ratio of boys to girls was 7:1.

Conclusions: The prevalence of autistic spectrum disorder in a geographically based population employing two source capture-recapture analysis is comparable to that quoted for the best active ascertainment studies. This technique offers a tool for establishing the prevalence of this condition in health service populations to assist in planning clinical services.
\end{abstract}

$\mathrm{T}$ he clinical picture of autism is modified by age and intellect but always encompasses the triad of impairments in speech and language, social cognition, and imaginary thought. ${ }^{1}$ Diagnostic classifications such as the International Classification of Diseases, 10th edition (ICD-10) ${ }^{2}$ and the Diagnostic and Statistical Manual, 4th edition (DSMIV) ${ }^{3}$ categorise resulting behaviours into diagnostic groups such as autism, Asperger's syndrome, atypical autism, and pervasive developmental disorder-not otherwise specified (PDD-NOS). ${ }^{4}$ Increasingly these are considered an autistic spectrum and although debate continues, ${ }^{5}$ this spectrum disorder concept and resulting variety of special needs may aid service planning. ${ }^{6}$

Autistic spectrum disorders (ASD) are now considered probably the commonest serious childhood developmental disability. ${ }^{7}$ Concern surrounds a perceived rising prevalence and whether it is due to greater diagnostic acumen and wider diagnostic labelling or underlying environmental/medical causes. ${ }^{8}$ The latter concerns parents and has fuelled debates on issues such as putative links with the MMR vaccine. ${ }^{9}$ The cause of ASD is probably multifactorial and no unifying aetiology has been established. ${ }^{10}$

While active case ascertainment should provide the most accurate prevalence figures, there are practical difficulties and no satisfactory screening instrument. ${ }^{11}$ Surveillance systems and patient databases are valuable and cost-effective epidemiological tools, but are limited by under-ascertainment. Potentially where two comprehensive databases cover a geographical population, the capture-recapture technique ${ }^{12}$ may be used to estimate the true population and likely completeness of current systems. This is particularly significant for conditions like ASD, with ill defined onset and varying severity. Capture-recapture techniques may offer efficient realistic alternatives to population screening. ${ }^{13}$ We proposed to establish the point prevalence of ASD in under 15 year olds in Lothian using all relevant health service datasets, and additionally calculate estimated true prevalence using the capture-recapture technique developed in biological research ${ }^{10}$ and successfully employed in studies of eye disorders $^{14}{ }^{15}$ and traffic injuries. ${ }^{16}$

Lothian is an ideal setting, having established comprehensive local child development diagnostic clinics linking to a specialist communication clinic. Professionals working with ASD enjoy strong interdisciplinary links in a combined children's service and collaborate via a Professional Interest Group, supported by the National Autistic Society. Furthermore, a multidisciplinary forum of Lothian education authorities reviews children with suspected ASD and educational support requirement.

We hypothesised that this collaborative climate would enable collection of data on children with an ASD and estimation of both reported and "true" prevalence of ASD using the capturerecapture technique. The importance of identifying the prevalence of ASD is well documented, ${ }^{6}{ }^{17}$ being a lifetime disorder with implications for many services. We anticipated that our results could be extrapolated to these services.

\section{METHODS}

Age, gender, and postcode sector were collected for all Lothian children with an ASD aged under 15 years, born between 1 July 1986 and 30 June 2001.

The second author (A E O'Hare) is the consultant in Community Child Health responsible for strategic planning of paediatric communication disorder services in Lothian. Lothian Universities NHS Trust's Caldicott guardian granted permission to access appropriate datasets under strict 
confidentiality and security measures. Nine datasets containing information on ASD cases were identified and questionnaires sent to dataset holders ascertaining catchment area, computerisation, data retention/removal, referral process, inclusion criteria, and definitions of ASD conditions used. Children with autism, ASD, atypical autism, Asperger's syndrome, and pervasive developmental disorders were included. Only Child and Family Mental Health services who had lost funding for dataset maintenance did not provide information. Other datasets would probably capture these children; as copy correspondence on such children would go to the Community Child Health Department, this could not be confirmed. Only four datasets were computerised.

The diagnosis dataset covered the previous 10-15 years' diagnostic clinics throughout Lothian. Diagnoses were based on ICD-10 or DSM-IV pervasive developmental disorders, made using observational assessment by the senior paediatrician or child psychiatrist, with evaluation of communication, reciprocal social interaction, and repetitive behaviours. Attention was paid to the child's ability to attend, imitate, comprehend, and use language, play appropriately with toys, and interact socially. A range of assessment tools including the Childhood Autism Rating Scale (CARS), ${ }^{18}$ Gilliam Autism Rating Scale, ${ }^{20}$ and Autism Diagnostic Observation Schedule (ADOS $)^{21}$ were also employed. Training and practice of senior clinicians using these formal assessments differed throughout Lothian. A E O'Hare reviewed medical notes for all cases to ensure that a senior paediatrician or psychiatrist had made the appropriate diagnosis.

The diagnostic clinic dataset captured children reviewed in child development clinics at secondary and tertiary levels specialising in communication disorder assessment. Diagnosis were usually based on ICD-10 criteria (the coding system for inpatients), but sometimes by DSM-IV (the clinician's preference). Most children with an ASD received their diagnosis in these clinics, although occasionally in Neurology or Child and Family Mental Health Service Departments. Children seen in the latter were often crossreferred to the Child Development Communication Clinic, especially those of preschool age.

Four further datasets were Lothian-wide. The largest, the Special Needs System, holds information on children with complex disability defined as needing access to two specialised or second tier services within a range of providers including health, education, social, or voluntary services. Diagnosis on the Special Needs System was based on ICD-10 criteria. The pragmatic category of ASD, broadly coinciding with pervasive developmental disorder and including autism and Asperger's syndrome, described children on these datasets. Essentially, a diagnosis is clinical judgement guided by behavioural symptoms, and ASD is increasingly accepted as encompassing children having significant impairments in communication, social understanding, skill/flexibility in thinking, and behaviour, giving rise to special needs. ${ }^{17}$ Families can opt out of this system. The remaining datasets contributed to prevalence calculations but were either not Lothian-wide, or targeted narrower age groups or particular clinical needs.

The Lothian Autistic Society, a voluntary organisation, held the final Lothian-wide dataset, and distributed a letter about the study inviting members to provide information if they wanted their child to be included.

Data analysis and statistical tests were conducted using Stata, version 8.2.2. All children from these datasets were included in the prevalence calculation. Point prevalence was used as the estimate was calculated on a specified date, and ASD, despite being of ill defined diagnosis and onset, is stable and lifelong. The total population of under 15 year olds resident in Lothian in mid-2001 was 134 661, based on 2001 population estimates by single age. ${ }^{23}$
Prevalence calculation for a point estimate: ${ }^{24}$

- $\mathrm{P}=$ number of existing cases of a condition/total population

The likely true number of Lothian children with an ASD was estimated using the two source capture-recapture technique on the best two sources. In this adaptation of the biological sampling capture-recapture technique, databases replace captures, and cases common to both sources represent "tagged" specimens (those captured twice). Four assumptions underpin the two source capture-recapture technique. ${ }^{15} 1625$ Firstly, two comprehensive datasets in a "closed" population (constant population during the study period) must be used. The chance of being referred onto either database must be equal, and datasets must be relatively independent (databases do not refer cases to each other). Finally, cases must be matched confidently and accurately between sources. In practice the two datasets must include all ages of children under 15 years old and cover all of Lothian. Both the diagnostic dataset and Special Needs System satisfied this.

In a two source capture-recapture technique on databases, the "true" number of cases $(\mathrm{N})$ is calculated as: ${ }^{12} 1525$

- $\mathrm{N}=[(\mathrm{X}+1)(\mathrm{Y}+1) /(\mathrm{Z}+1)]-1$

where:

$\mathrm{X}=$ number of cases from source 1

$\mathrm{Y}=$ number of cases from source 2

$\mathrm{Z}=$ number of cases common to both sources.

A confidence interval is calculated as: ${ }^{12} 2526$

- $\mathrm{N} \pm 1.96[\sqrt{ } / \operatorname{Var}(\mathrm{N})]$

where $\operatorname{Var}(\mathrm{N})=\left[(\mathrm{X}+1)(\mathrm{Y}+1)(\mathrm{X})(\mathrm{Y}) /(\mathrm{Z}+\mathrm{l})^{2}(\mathrm{Z}+2)\right]$.

The estimated total number of cases can be used in an alternative prevalence estimate of complete ascertainment using the same denominator. ${ }^{23}$

The apparent trend of smaller proportions of children with ASD in older age groups was tested formally using an extension of the Wilcoxon rank sum test for trend. Children aged less than 3 years $(n=7)$ were excluded; this age group are least likely to be diagnosed with ASD as the condition is more commonly detected once children enter formal education. State funded nursery is available for those aged over 3 years.

\section{RESULTS}

A total of 443 children were identified with ASD (mean age 8.4 years, range $2.5-15$ years). Age distributions of children

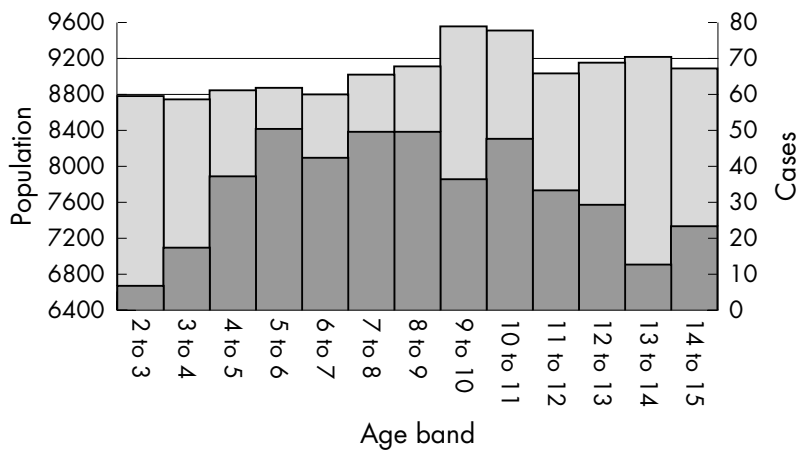

Figure 1 Background population (pale grey) and number of children with an ASD (dark grey) in Lothian for each age band included in the study. 
with an ASD and the background Lothian population are shown in fig 1 . There appeared proportionately reduced numbers of children with ASD in the youngest and oldest ages compared to those aged between 4 and 10 years. There was a significant trend of decreasing proportions of children diagnosed with ASD with increasing age $(p=0.003)$.

There were 369 boys and 53 girls ( 11 no data). Table 1 details median age and male:female ratios in each dataset. The median age for Spectrum and SureStart data reflect that these are "early intervention" services for preschool children. The male:female ratio for the Special Needs System was 7:1, but there was an increased ratio of 12.1:1 for the Learning Disability Community Nursing Team service.

Using 443 cases and the denominator of $134661,{ }^{23}$ prevalence of ASD in Lothian is 32.9 per 10000 children (95\% CI 29.8 to 36.0 ).

A total of 243 and 268 children with ASD were recorded on the Special Needs System and diagnosis datasets respectively; 109 of these were common to both. An estimated total of 596 children with ASD in Lothian was calculated, using the two source capture-recapture calculation on these two sources.

- $\mathrm{N}=[(243+1)(268+1) /(109+1)]-1=595.7$

(95\% CI 532.4 to 658.9$)$

Based on the estimate of 596 children with an ASD using the same denominator yielded an estimated prevalence of 44.2 per 10000 children (95\% CI 39.5 to 48.9 ) can be calculated. This is arguably the true prevalence if complete ascertainment of cases was achieved.

- $596 / 134661=0.00442 \times 10000=44.2$

\section{DISCUSSION}

We established a point prevalence of 32.9 per 10000 for children with ASD in Lothian, Scotland, comparable to those quoted by Honda and colleagues ${ }^{27}$ and Powell and colleagues. ${ }^{28}$ Although the Honda et al study used direct ascertainment, it was based on a small population, had wide confidence intervals, and studied childhood autism without widening the concept to ASD. The Powell et al study used a larger population and similar approach to this study. We therefore consider that our prevalence figures are likely to be the minimum for childhood ASD and plans for services are likely to be inadequate if provision is for less than this.

However, this study aimed to estimate what "true" prevalence might be, assuming the possibility that not all children were captured on the nine datasets. By applying the "capture-recapture" technique for the first time in ASD, the estimated point prevalence was considerably higher at 44.2 per 10000 . The resulting confidence intervals overlap with the active ascertainment study by Baird and colleagues ${ }^{11}$ and approach the lower limits of the Chakrabarti and Fombonne estimate. ${ }^{29}$ The former reported on the prevalence of children with ASD, and the latter pervasive developmental disorder (PDD), although it is likely that similar children were included. In Lothian the term ASD has largely replaced PDD and encompasses autism, Asperger's syndrome and atypical autism. It could be argued that ASD is too inclusive a concept, leading to inflated prevalence figures. However, children in this study were known to services and therefore likely to have significant special needs. It is probably more realistic to plan services for this broader group of children whose central difficulties are in social communication and cognition. Active ascertainment studies such as that in a Welsh education authority have shown a minimum prevalence of 20.2 per 10000 for children with ASD in mainstream schools; many of these children were unknown to specialist services and had unmet needs for further assessment and management. ${ }^{30}$

Comparison of our two prevalence estimates suggests that only $74 \%$ of children with ASD were known to services (identified by any dataset). The age distribution might support the contention that this shortfall was primarily for younger and older children as prevalence across the age groups of 4-12 years was steady. Few children under 3 years old received a diagnosis, consistent with published experience that although parents often retrospectively recognise noticing abnormal interaction and communication in their infant, they had either not sought assessment or had been reassured. ${ }^{31}$ The relative lack of older children may be an artefact of diagnostic practice or they may have graduated out of paediatric services. The 13-15 year olds would have received their MMR vaccination as data collection was completed by July 2001. The children's dates of birth were between July 1986 and June 2001; the MMR was introduced in Lothian in 1987, yet they are less numerous than 4-10 year olds. This would be against an MMR trigger but consistent with greater recognition and acceptance of ASD or better recording as recently suggested. ${ }^{9}$

The "capture-recapture" assumptions must be considered possible explanations for differences between reported and estimated prevalence. Firstly, the "closed" population assumes families are not immigrating/emigrating during the study period. However, estimations suggested Lothian's population increased by $3.7 \%$ between 1991 and $1999 .{ }^{23}$ Secondly, children could be accurately matched between datasets; this study matched on initials, gender, date of birth, and postcode, which should satisfy this assumption. Thirdly, children must have equal chance of referral onto either dataset. Doctors and health visitors refer onto both, although health visitor "concern" may be channelled through the child's general practitioner, speech therapist, or paediatrician for diagnostic clinic referral. Finally, the two systems must be independent of each other. Although there is overlap in referrers, the systems do not automatically refer to each other. There is a degree of dependence of the systems. However, most children are referred to the Special Needs

\begin{tabular}{|c|c|c|c|c|c|c|}
\hline Dataset & $\mathrm{N}$ & $\begin{array}{l}\text { Median } \\
\text { age }\end{array}$ & Range & Min & Max & M:F ratio \\
\hline Special Needs System & 243 & 8.4 & 12.4 & 2.6 & 15 & $7.1: 1$ \\
\hline Diagnosis dataset & 268 & 7.3 & 12.5 & 2.5 & 15 & $6.2: 1$ \\
\hline Learning Disability Community Team & 40 & 9.6 & 10.5 & 4.1 & 14.6 & 12.3:1 \\
\hline Lothian Autistic Society & 82 & 8.4 & 11 & 2.9 & 13.9 & $6.5: 1$ \\
\hline Royal Hospital for Sick Children (hospital dataset) & 58 & 8.2 & 12.2 & 2.6 & 14.8 & $8.7: 1$ \\
\hline Speech and Language Therapy & 61 & 9.6 & 12.2 & 2.6 & 14.8 & $6.6: 1$ \\
\hline VTSS (school age children) & 9 & 9.2 & 9 & 6.5 & 13.8 & N/A* \\
\hline Spectrum (preschool children) & 21 & 3.9 & 2.7 & 2.6 & 5.3 & $8.1: 1$ \\
\hline SureStart & 54 & 4.7 & 3.9 & 2.6 & 6.5 & $9.8: 1$ \\
\hline
\end{tabular}




\section{What is already known on this topic}

- Autistic spectrum disorders (ASD) are no longer considered rare conditions; they may be the most common serious developmental disabilities of childhood. Active case ascertainment methods-that is, screening, should provide the most accurate prevalence figures by identifying all affected children, but have many practical difficulties including a lack of an adequate screening instrument

- Capture-recapture techniques have been adapted for use in database based epidemiological studies to estimate a "true" population size; these methods have been used successfully in other conditions

\section{What this study adds}

- The point prevalence of ASD in Lothian using passive ascertainment was 32.9 per 10000 . This prevalence estimate should be considered the minimum rate when planning services. Using the capture-recapture to estimate the total number of children with an ASD, the prevalence estimate was 44.2 per 10000

- The capture-recapture technique is a useful tool for establishing the true prevalence of ASD in health service populations for service planning

System by health visitors. At this point the child is not diagnosed, but problems they are encountering are documented. Once the child is reviewed and formally diagnosed with ASD, the Special Needs System is updated. Theoretically a child might be seen in the interim by a diagnostic clinic paediatrician and also referred onto the Special Needs System, but this would be uncommon. Therefore we consider that the assumptions are reasonably well met.

The male to female ratio of 7.1:1 compares with other studies. ${ }^{82832}$ The high proportion of males in the Learning Disability Community Nursing service reflect inclusion of children with severe challenging behaviour due to a combination of autism and cognitive impairment. ${ }^{33}$

In conclusion, the prevalence of autistic spectrum disorder in this large geographically based population employing a two point "capture-recapture" analysis compares to those of active ascertainment studies. The technique offers a tool to establish the prevalence of these conditions in health service populations for service planning.

\section{Authors' affiliations}

M J Harrison, H Campbell, Dept of Public Health Sciences and Child Life and Health, Reproductive and Developmental Sciences, University of Edinburgh, UK

A E O'Hare, A Adamson, J McNeillage, Royal Hospital for Sick Children, Edinburgh, UK

Competing interests: none declared

\section{REFERENCES}

1 Wing L, Gould J. Severe impairments of social interaction and associated abnormalities in children: epidemiology and classification. J Autism Dev Dis 1979;9:11-29

2 World Health Organisation. The International Classification of DiseasesClassification of Mental and Behavioural Disorders Diagnostic Criteria for Research (ICD-10), 10th edn. Geneva: WHO, 1993.

3 American Psychiatric Association. Diagnostic and Statistical Manual for Mental Disorders (DSM-IV), 4th edn. Washington: APA, 1994

4 Baird G, Cass H, Slonims V. Diagnosis of autism. BMJ 2003;327:488-93.

5 Medical Research Council. Review of autism research: epidemiology and causes. Medical Research Council, 2001

6 Public Health Institute of Scotland. Autistic spectrum disorders: needs assessment report. NHS Scotland, 2001.

7 Gillberg C, Wing L. Autism: not an extremely rare disorder. Acta Psychiatr Scand 1999;99:399-406.

8 Webb EV, Lobo S, Hervas A, et al. The changing prevalence of autistic disorder in a Welsh health district. Dev Med Child Neurol 1997;39:150-2.

9 Lingam R, Simmons A, Andrews N, et al. Prevalence of autism and parentally reported triggers in a north east London population. Arch Dis Child 2003;88:666-70.

10 Szatmari P. The causes of autism spectrum disorders. BMJ 2003;326:173-4.

11 Baird G, Charman T, Baron-Cohen S, et al. A screening instrument for autism at 18 months of age: a 6-year follow-up study. J Am Acad Child Adolesc Psychiatry 2000;39:694-702.

12 Hook E, Regal R. Capture-recapture methods in epidemiology: methods and limitations. Epidemiol Rev 1995; 17:243-64.

13 Laporte RE. Assessing the human condition: capture-recapture techniques. BMJ 1994;308:5-6.

14 Campbell $H$, Holmes E, MacDonald S, et al. A capture-recapture model to estimate prevalence of children born in Scotland with developmental eye defects. J Cancer Epidemiol Prev 2002;7:21-8.

15 Rahi J, Dezateux C. Measuring and interpreting the incidence of congenital ocular anomalies: lessons from a national study of congenital cataracts in the UK. Invest Opthalmol Vis Sci 2001;42:1444-8.

16 Morrison A, Stone DH. Capture-recapture: a useful methodological tool for counting traffic related injuries? Inj Prev 2000;6:299-304.

17 Le Couteur A. National Autism Plan for Children (NAPC). National Initiative for Autism Screening and Assessment (NIASA), 2003.

18 Dilalla DL, Rogers SJ. Domains of the Childhood Autism Rating Scale: relevance for diagnosis and treatment. J Autism Dev Disord 1994;24:115-28.

19 Schopler E, Reichler RJ, DeVellis RF, et al. Toward objective classification of childhood autism: Childhood Autism Rating Scale (CARS). J Autism Dev Disord 1980;10:91-103.

20 Gilliam JE. Gilliam Autism Rating Scale (GARS). PRO-ED, 1995

21 Lord C, Risi S, Lambrecht L, Cook EH Jr, et al. The autism diagnostic observation schedule-generic: a standard measure of social and communication deficits associated with the spectrum of autism. J Autism Dev Disord 2000;30:205-23.

22 Stata Corp. Stata statistical software. College Station, TX: Stata Corporation, 2001.

23 General Register Office for Scotland. Mid-2001 population estimates, 2002. http://www.gro-scotland.gov.uk/grosweb.nsf/pages/file4/\$file/ 2001 mpesya.xls.

24 Hennekens $\mathrm{CH}$, Buring JE. Epidemiology in medicine. Lippincott Williams \& Wilkins, 1987.

25 International Working Group for Disease and Forecasting. Capturerecapture and multiple-record systems estimation. II: applications in human diseases. Am J Epidemiol 1995;142:1059-68.

26 Regal R, Hook E. Goodness-of-fit based confidence intervals for estimates of the size of a closed population. Stat Med 1984;3:288-91

27 Honda H, Shimizu Y, Misumi K, et al. Cumulative incidence and prevalence of childhood autism in children in Japan. Br J Psychiatry 1996;169:228-35

28 Powell JE, Edwards A, Edwards $M$, et al. Changes in the incidence of childhood autism and other autistic spectrum disorders in preschool children from two areas of the West Midlands, UK. Dev Med Child Neurol 2000;42:624-8.

29 Chakrabarti S, Fombonne E. Pervasive developmental disorders in preschool children. JAMA 2001;285:3093-9.

30 Webb E, Morey J, Thompsen W, et al. Prevalence of autistic spectrum disorder in children attending mainstream schools in a Welsh education authority. Dev Med Child Neurol 2003;45:377-84.

31 Gray KM, Tonge BJ. Are there early features of autism in infants and preschool children? J Paediatr Child Health 2001;37:221-6.

32 Kadesjo B, Gillberg C, Hagberg, B. Brief report: autism and Asperger syndrome in seven-year-children: a total population study. J Autism Dev Disord 1999;29:327-31.

33 Mental Health Foundation. Don't forget us: children with learning disabilities and severe challenging behaviour. Mental Health Foundation, 1997. 